Cite: Chalip, L., Green, B.C., Taks, M., \& Misener, L. (2017). Creating sport participation from sport events: Making it happen. International Journal of Sport Policy and Politics. 9, 257-276. DOI: 10.1080/19406940.2016.1257496.

\title{
Title: Creating Sport Participation from Sport Events: Making It Happen
}

\begin{abstract}
Sport participation is a claimed benefit of elite sport events, but the facts do not support that claim, and means to capitalize upon events in order to build participation have yet to be developed. Through a combination of dialectical inquiry, brainstorming and nominal group, 12 expert panellists were invited to consider the challenges, opportunities, and prospects of leveraging sport events to enhance sport participation at local levels. The model consists of three elements: (1) the hierarchical nature of the context (culture; opinions and attitudes; systems and structures), (2) three types of organizations with a stake in the leveraging process (event, sport, and non-sport entities), and (3) resources needed (human, physical, and knowledge). The centre reflects the core, which is sport participation. A series of strategic questions are presented to guide formulation and implementation of strategies and tactics for leveraging sport events to build sport participation. The findings are consistent with sport development outcomes from the 1984 Los Angeles Olympic Games. It is concluded that sport events can be leveraged to enhance sport participation if the necessary alliances among sport organizations, event organizers and non-sport stakeholders are forged to integrate each event into the marketing mix of sport organizations. It is also noted that potential barriers to enhanced participation need to be addressed, particularly lack of available capacity to absorb new participants, crowding out of local participation by the event, and the disincentives resulting from elite performances that seem outside the reach of aspiring participants.
\end{abstract}

Keywords: Sport events; Leverage; Legacy; Sport participation; Sport development; LA84 Foundation; Los Angeles Olympic Games 


\section{Introduction}

Sport events can be expensive to host, and even to bid for (Chalip \& Heere, 2014). The traditional justification for communities to seek and host events has been that the overall economic impact can make the effort worthwhile (Mules \& Faulkner, 1996). However rigorous economic evaluation suggests that hosting a sport event can cost an economy more than it gains, even when more simple economic analyses suggest otherwise (Taks et al., 2011). In addition, sport events can also engender an indefensible income transfer among local citizens (Putsis, 1998). Consequently, there has been an ever-increasing effort to find intangible but arguably valuable outcomes from hosting sport events.

More recently, there has been substantial interest in the ways that nation states use sport events to advance their international status and reputations through the soft power associated with hosting large sport events (Grix \& Brannagan, 2016). In point of fact, the objectives from so doing vary as a function of the different needs that are surmised by political leaders in different countries (Attali, 2016; Carter, 2016; Grix \& Houlihan, 2014). Although expectations for the political value of sport events are clear from this work, whether those benefits are obtained or sustained is less clear. In the ongoing maelstrom of international politics, the soft power gains from sport may be limited, but nonetheless have value with reference to how a host country sees itself (Chalip, 1987), and therefore they may have value for nation building (Hong, 2011; Koch, 2013).

Pursuant to that end, enhanced sport participation has been advanced as one of the significant benefits that might accrue from hosting sport events. For example, building sport participation was an explicit promise in the bids for the 2012 London Olympic Games (Girginov \& Hills, 2008) and the 2008 Beijing Olympic Games (Dong \& Mangan, 2008); it was a feature of planning for the Toronto Pan American Games (Misener, 2013); it has been a core concern for 
Brazil as a mega event host (Reis, Rodrigues de Sousa-Mast, \& Gurgel, 2013). The expectation has been that sport events will inspire those who watch to emulate the athletes they see, at least to the extent of engaging in sport.

The problem with that expectation is that it is inconsistent with facts as we know them. One of the earliest tests of that expectation (Hindson, Gidlow, \& Peebles, 1994) found that only six of thirty-five New Zealand sport clubs had any discernable increase in enquiries following the Albertville and Barcelona Olympic Games, and only five clubs claimed even a slight increase in actual membership - levels too low to suggest any positive effect from the Olympic Games. In Australia, more Olympic sports showed declines in participation after the Sydney Olympic Games than experienced gains (Veal, 2003). Subsequent analysis suggested that although elite sport in Australia may ultimately have benefited from the Sydney Olympic Games through enhancements to the sporting infrastructure, the gains occurred at the expense of mass sport participation (Toohey, 2008). A British study of participation after the Manchester Commonwealth Games found that although people claimed to be doing more sport as a result of the Games, membership in sport clubs throughout Greater Manchester actually declined (MORI, 2004). Similarly, although the gold medal for curling won by the British women's team at the Salt Lake City Olympic Games may have resulted in a small increase in the amount of participation by those already active in the sport, it had no discernible effect on the numbers participating (SportScotland, 2004). There may have been a short-term increase in participation in a few sports among Brazilians after the 2007 Pan American Games, but there was no sustained increase (Reis, Rodrigues de Sousa-Mast, \& Gurgel, 2013). The conclusion to be drawn from these findings is that sport events, especially those at which elite athletes perform, do not prompt people to participate, although some of those who do participate may increase their amount of participation for a short time. 
Despite consistent findings to the contrary, there seems to be ongoing hope for a positive effect from sport events on participation. For example, in the lead-up to the London Olympic Games, the legacy plan boasted, "We will inspire people to make sport happen at the local level, embedding the Olympic and Paralympic values in grassroots sport" (Department for Culture, Media and Sport, 2010, p. 4). However, promises of this kind create expectations that can become a source of scorn when they are not achieved. For example, writing in the Guardian almost three years after the London Olympic Games, Gibson (2015) described a strong sentiment that the public had been misled by promises of added sport participation consequent on those Games. He pointed out that participation had actually dropped, especially among the poor and working classes, and he suggested that new debates in parliament about promoting sport participation demonstrated how much the promises had been little more than idle rhetoric.

Close analysis of the actual effects of elite sport events suggests why this may be the case. In the first place, the high profile of medal-level performances at elite sport events encourages governments to invest in elite sport in order to foster international prestige, positive sentiments among the populace, and a concomitant rise in participation (Grix \& Carmichael, 2012). Since budgets are finite, the consequent investments in elite sport come in lieu of resources to build mass sport participation (Brookes \& Wiggan, 2009; Green, 2007; Toohey, 2008). Governments might dismiss these effects on the grounds that the positive sentiments about successful athletes will nonetheless foster participation (Grix \& Carmichael, 2012). However, elite sporting achievements have the potential to discourage sport participation because viewers perceive a substantial gap between their capabilities and those of elite athletes (Hindson, Gidlow, \& Peebles, 1994). Further, watching elite sport is itself an attractive but passive leisure activity, so watching elite sport may work against sport participation because it is a distraction from doing sport (Veal, 2003). 
Given that elite sport events continue to be promulgated, and enhancement of sport participation remains a claimed benefit of such events, the emergent concern has been to identify means to capitalize upon events in order to build participation. Physical educationists in Brazil expect that hosting mega events will only be effective if plans are developed and implemented for building sport participation (Reis, Rodrigues de Sousa-Mast, \& Gurgel, 2013). Girginov and Hills (2008) argued that events like the Olympic Games might be used to build participation, but only if there is a concerted effort to do so. They contend that (1) events must be used to build the capacity of sport providers, (2) the public should participate in planning and implementing programs to capitalize on events, and (3) there should be procedures for sport providers to learn from their efforts to build participation using events. The consequent challenge is to formulate and refine a model for using events to build participation. This study describes such a model.

The use of expert creative imagination to build strategy is well established in business (Levitt, 1983; Spender, 2014), the military (Hart, 1991), and academic study (Costa, 2005). An array of group decision methods has been developed for that purpose (Chalip, 2001). Ritchie (2000) demonstrated the value of using such techniques when considering the potentials for legacy from a sport event. This study employs group decision methods among a group of experts to formulate and elaborate a model for using events to build participation. The model is then compared to processes and outcomes that led to a concerted effort to use the Los Angeles 1984 Olympic Games to build sport in southern California (Wilson, 2015). That event was chosen for purposes of comparison because it remains the only mega-sport event from which a sustained sport development infrastructure was created. The Los Angeles case is incorporated to explore the feasibility of leveraging events for sport participation through comparison of the model to an avowedly successful example. 


\section{Method}

The Expert Panel

This research involved multiple methods with a panel of experts over the course of a strategic development and assessment workshop. Panellists were identified and invited to participate in a day long workshop to consider the challenges, opportunities, and prospects of leveraging sport events to enhance sport participation at local levels. The panel was comprised of Canadian 12 experts from a variety of organizations that would (or could) be involved in (and benefit from) leveraging sport events for participation and development. The panel consisted of two marketers, two public school representatives, two event experts, three (public sector) sport executives, one parks and recreation executive, one university sport administrator, and one community development specialist. The 12 experts were assigned by the research team to one of three smaller groups ensuring a balance of expertise within and between groups. A dialectical inquiry process (Mitroff \& Betz, 1972) was used throughout the workshop, as this technique has been shown to provide better strategic results than other related techniques. This was complemented by brainstorming and nominal group technique in the small group settings and when the group met as a whole. All sessions were enabled by the use of facilitators trained in the dialectical inquiry techniques of including brainstorming and nominal group formats. Break-out sessions were guided by one trained facilitator with members of the research team serving as recorders for each group.

The day began with an introduction to leveraging for participation based on previous work (Taks, Green, Misener, \& Chalip, 2014; Taks, Misener, Chalip, \& Green, 2013). Two break-out sessions were conducted in the morning; the first asked panellists to consider what was currently available to leverage events for participation (i.e., opportunities, resources, and points of leverage), 
the second asked each group to consider barriers and constraints. Each small group breakout session began with nominal group technique (cf. Harvey \& Holmes, 2012) in which participants were asked to write down independently their ideas in relation to the topic. Each participant was then asked to contribute one idea at a time on a rotating basis until all original ideas had been introduced and recorded in view of all group members. This portion was followed by a brainstorming session to build on the ideas. All groups then convened to share their results and discuss ideas with the full group. The groups chatted informally over lunch, then the break-out groups repeated the morning procedures based on two new prompts: (1) How we might proceed to leverage events for participation (i.e., strategies and tactics), and (2) How can we make it happen (i.e., implementation). All groups presented their ideas to the full group at the end of the day, which was followed by a group discussion. The research team recorded the outcomes of each step in the process.

The notes from all sessions were content analysed by all members of the research team, and an inventory created of all unique comments and ideas from the four sessions: (a) opportunities, resources, and points of leverage, (b) barriers and constraints, (c) strategies and tactics, and (d) implementation. The members of the research team then started to identify relevant themes by grouping similar items from the inventory through a brainstorming process. Subsequently each comment or idea was assigned to the relevant themes. Similar comments or ideas could be attributed to different themes. The research team continued this process until all of the ideas and comments were exhausted. This process then led to the development of a model for leveraging sport events for sport participation and development. The model, including a description and a set of matrices based on the content analysis, was sent to all workshop participants for comment. All comments were incorporated into the final model. 
Comparison of Findings with the Los Angeles Olympic Games

Next, studies of the 1984 Olympic Games in Lost Angeles were examined, as were the website and reports of the sport development organization that was created after those Games, which is now called the LA84 Foundation. Newspaper coverage from the Los Angeles Times from 1983-1986 was also examined to obtain a contemporary perspective on establishment of the Foundation. Information from those materials was then compared to the model derived from the experts.

\section{Results}

The Expert Panel

The model, which is illustrated in Figure 1, consists of three elements: (1) a series of concentric circles representing the hierarchical nature of the context, (2) three slices representing the three types of organizations with a stake in the leveraging process, and (3) three rays cutting across the layers and slices representing categories of resources needed to enable leverage. The centre of the model reflects the core of the leveraging effort: the sport participation goals of three types of organizations (the event, the sport, and non-sport entities). It is important to note that the sport participation goals can be driven by any one of these entities or can be shared goals. However, the goals must, at minimum, be in alignment with one another. The goals are context specific and are expected to vary based on the type and scope of the event, as well as the community and geographic context of the event.

The panellists discussed three types of organizations as distinctively important for sport participation: sport organizations, event organizers, and non-sport organizations (that could play a part in creating or supporting sport participation). Panellists felt it was unlikely that any one organization or one type of organization could leverage effectively. Together these three sets of 
organizations constitute the community necessary to leverage an event to build sport participation. The core challenge of formulating goals and means to reach those goals originates at this aggregated community level. So, the first challenge is to engender the necessary impetus for goal setting from which everything else follows. A core group of interested parties must be formed in order to enable that to occur. This is fundamentally a collective action challenge and, as with other collective action challenges, a group of social entrepreneurs must self-identify and come together for this purpose (Medina, 2009). That is a precondition for the model to be made effective.

With the formation of a collective community, all other aspects of the model affect the strategies and tactics to support the goals: the systems and structures (of the event, sport, and nonsport entities); as well as the resources (physical, human, and knowledge-based) that are mobilized to implement the strategies and tactics. It is important to note that these resources are three dimensional, indicating that the resources (can) pertain to each entity, or even overflow entities (i.e., event, sport, and non-sport). The concentric circles represent the hierarchical nature of the context. Structures and systems are developed, accepted, and sustained in response to attitudes and opinions, which are in turn influenced by the broader cultural values in which they are embedded. Each of the contextual factors represented by the concentric circles both affects and is affected by the resources available - physical, human, and knowledge-based resources. Thus, these resources are represented as rays that cut across and interact with each level of the model, but also with each entity. At each layer of the context (culture, attitudes and opinions, structures and systems), challenges and strategies were identified for each of the three types of organizations (event, sport, and non-sport). Then, resource challenges were identified for each organization type. As the inner layers are embedded in those surrounding them, the analysis begins at the outermost layer with an examination of the strategic issues arising from culture. 


\section{Culture}

The culture in which the community is embedded has a significant impact on the attitudes and opinions of members of that community, the goals that are to be obtained, the manner in which problems are framed, and the strategies that are considered. The panellists identified challenges related to overall cultural values and beliefs, as well as cultural beliefs about sport and sport events. For example, cultural beliefs have been shown to be related to sport participation levels, particularly the participation of girls and women as well as to the appropriateness of participation in a specific sport (Chalabaev et al., 2013),. Panellists were concerned that community groups would be difficult to reach via sport events due to the low cultural value of sport. On the other hand, sport and sport events have high cultural currency for many groups (Cashman, 2010; Jay, 2013). In fact, sport-for-development organizations routinely use sport as a draw to entice underserved and underrepresented youth to partake in non-sport services (Green, 2008). Thus, panellists felt that culture, particularly cultural values and beliefs could be both a challenge and an opportunity when leveraging events to build sport participation.

The panellists identified the media as a significant shaper of cultural values and beliefs. Like the cultural value of sport, media influences were seen as both an opportunity and a potential challenge. Media decisions regarding what will be broadcast and how the event, sport, and athletes will be framed greatly influence perceptions of the event, the sport, and its athletes. Mega-events can take advantage of the media's focus, but smaller or less popular sport events may find it difficult to attract media attention. Consequently, these events are seen as less culturally relevant. Although media attention is generally assured for mega-events, its value in leveraging for participation is unclear. Media focus on mega-events and narratives built around elite athletes that participate in these events may create an unexpected challenge. If these events are to encourage 
sport participation, non-participants need to connect to the athletes, the sport, and/or the event. Yet, the more media narratives focus on the superhuman feats of skill and the athletes' abilities to overcome every challenge, the more difficult it is for the average spectator to identify with them. Therefore, they are unlikely to see these athletes as role models or to be inspired to participate in sport. Instead, the disconnect between themselves and the athletes participating in the event is strengthened.

Alternative strategies were also suggested. One alternative strategy identified is to influence the narratives themselves. Rather than present the athletes as superhuman, provide narratives that show the athletes in ways that humanize them and help the audience to see that they, too, could enjoyably participate in the sport with some success. Parallel events or road shows can further reduce the gap by providing sport trials that include modified and/or mock events and associated festival activities. The key is to create a sense of efficacy -- that 'I can do that.'

Panellists also suggested alternatives to mainstream media - particularly communication strategies in which the message is controlled by the leveraging group itself. This can occur via messages delivered at the event itself, or via social media and other communication outlets controlled by the event and sport organization(s). A controlled message is important for both mega-events and non-mega-events, albeit for different reasons. The popular distinction between major (aka: 'real') sports versus minor (insignificant) sports may threaten leveraging opportunities for minor sports. In this case, social media may take the place of traditional media communications. Mega-events do not have trouble attracting media attention, but may still have difficulty in generating narratives that would facilitate sport participation and development. Narratives can be provided via the event and sport organizations' websites, blogs, and other social media sites. Twitter, Instagram, and other media can be used to deliver messages, to drive traffic to websites, 
and to facilitate the connection of fans (e.g., potential participants) and the sport, sport organizations, and the athletes (both elite and local).

Lastly, panellists suggested that the leveraging group create opportunities before, during, and after the event to raise awareness for the event and, more importantly, for participation opportunities in the community. Event organizers can also organize related symbolic events such flash mobs in public spaces, which can build awareness and intent. Offsite events in the pre- and post-event periods, particularly social events, could provide opportunities for potential participants to interact with athletes in ways that emphasize their ability to inspire while also highlighting their 'normalness' and similarity to potential participants.

In short, panellists felt that the success of leveraging efforts could potentially be both hindered and supported by the broader culture in which an event is embedded. Broader societal values were considered, and strategies suggested. However, significant culture change was expected to require a long-term effort. This effort begins with changes in individuals' attitudes and opinions. The necessary strategic questions to formulate that change are shown in Table 1.

\section{Attitudes and Opinions}

The workshop participants identified core attitudinal challenges facing event organizations as well as sport organizations. They did not readily identify attitude and opinion-based challenges for non-sport entities, perhaps due to the breadth and variety of these organizations. Three core challenges were identified: (1) attitudes and opinions about appropriate sport participation, (2) the gap between potential participants' views of themselves vis-à-vis event athletes, and (3) narrow attitudes of sport event personnel about ceremonies and festival.

The key challenges facing sport organizations as they attempt to leverage sport events straddle the line between culture and attitudes. This is not surprising, as attitudes and opinions are 
often shaped by culture. These two challenges are a function of (a) parents' attitudes and their influence on their children's sport participation, and (b) ethnic biases and presuppositions of sport administrators that steer children into (and away from) particular activities.

The easy answer to these challenges is a standard one: an educational campaign. This was also a ubiquitous suggestion from the panellists. Yet deeply embedded attitudes and beliefs rarely respond well to short-term education campaigns alone. In conjunction with educational campaigns, panellists suggested strategies to reach out to diverse populations to attend events where they can participate in fun sport-related games and contests, such as those provided at fan fests, and sport organizations can follow-up with attendees to arrange participation trials in their own neighbourhoods. By combining opportunities for trial with visits and interactions with athletes from varied ethnicities, genders, and social classes, sport organizations can begin breaking down entrenched attitudes and beliefs about 'proper' sport participation.

A broad attitudinal challenge to leveraging the event to encourage participation was the gap between potential sport participants' images of themselves and their beliefs about their own abilities in contrast to their image of the athletes and the athletes' abilities. For most, this gap is large and potentially intimidating. The challenge is to use the athletes as inspirational and aspirational figures to motivate sport participation. Panellists recommended that a felt sense of connection to the athletes should be engendered. This could include enabling encounters with athletes as well as creation of narratives about athletes highlighting their personal lives and challenges in ways that humanize them.

The last challenge is a function of the attitudes and opinions of sport and event planners, managers, and marketers. The panellists postulated that event workers generally fail to recognize the utility of ceremonies and festival (i.e., celebrations) as a tool to enhance sport participation 
(cf., Chalip, 1992). Instead, ceremonies and festival are confined to participant and audience experiences. Yet, these aspects of events have the capacity to incite interest in participation - either participation in the sport generally, or participation in the event itself. Panellists suggested that event managers should work to create symbolic links between the event (particularly the ceremonies and festival aspects), event audiences, and sport organizations. The strategic questions necessary to address attitudes and opinions are summarized in Table 2.

\section{Structures and Systems}

Panellists identified four challenges to leveraging events for participation that were thought to affect all three types of organizations. A lack of goal setting for sport participation and development was seen to undermine attempts to leverage. When an organization did set goals, there was rarely any alignment with (or interest in) the goals of other organizations active in event planning or implementation. Thus, the opportunity to work together towards a superordinate goal was lost. This challenge was seen to be exacerbated by two other challenges: (1) no organizations felt a sense of ownership or accountability to use events for sport participation and development, and (2) there were no existing structures to enable that leverage. A related challenge identified by the panel was conflicts of interest among stakeholder groups. In other words, the panel felt that there was little agreement regarding goals, strategies or implementation of leveraging tactics.

Crowding out effects were perceived to be a challenge facing both event organizers and sport organizations. Panellists saw challenges in these organizations' ability to leverage events for

sport participation that were a function of a crowded marketplace and the resulting competition for media attention, sponsorship and other funding, as well as facilities and human resources. Resource challenges will be discussed in a later section, however strategies and tactics to address the more general issue of crowding out are addressed here. 
Three strategies to address the lack of goal-setting were specified: (1) identify specific groups for target strategies (e.g., low income, seniors, single parents, immigrants), (2) develop a community sport development plan, and (3) choose events to fit with the agenda and goals for community sport development. Notice that these strategies require coordination among the organization types to be effectively implemented in service to shared, or at least complementary goals. Further, these strategies are unlikely to be successful without parallel strategies to address a lack of ownership and accountability, and a lack of structures to enable leverage. Although unstated, a separate or specific structure may be necessary to facilitate initial goal setting and planning as well as to inspire ownership of the goal, resultant strategies, and implementation tactics. It is difficult for existing organizations to take a leadership position relative to leverage as each is already working at full or near full capacity in their normal operations. For example, event organizers are focused on event operations; sport organizations on training their athletes for events; and non-sport entities are, by definition, focused on their own industry and customers.

Although panellists failed to envision strategies to build ownership and accountability for sport participation and development, the workshop sessions produced strategies regarding a potential structure to enable event leverage for this purpose. Panellists struggled to balance the need for leverage with organizations' existing foci on day-to-day operational concerns. Thus, their recommendations focused on creating new organizations/committees/task forces to oversee and create sustainable opportunities. They expected key stakeholders distinct from the event organizing committee to take ownership of these new structures and the sport participation and development agenda. The remit of this organization would include developing a community sport development plan and recommending events with the potential to enhance sport development in the community. By providing a voice, venue, and superordinate goal for stakeholders to coalesce 
to develop a community sport development plan, stakeholder conflicts could be minimized. An example of such a structure could, for example be a community sports council (Misener, Harmon, \& Doherty, 2013).

Sport participation was perceived to be an additional, albeit worthwhile, strategic initiative for events. Consequently, panellists felt that additional resources would be necessary to implement for sport participation leveraging. In addition to the specific committee responsible for leveraging, as discussed above, panellists suggested the need to access funding sources for social leverage through existing organizations or partnerships, or through yet another organization or committee akin to a sponsor of these particular efforts.

Two additional strategies emerged from the workshop sessions. Greater use of technology for knowledge mobilization was suggested by panellists as a way to better coordinate and communicate goals, strategies, and tactics for leverage. Panellists acknowledged the difficulty in organizing and attending face-to-face meetings for coordination. In response, they suggested webbased meeting and sharing tools such as video-conferencing, shared document sites, and web portals to connect sites. Partnerships were also recommended. Partnerships among event organizers, sport organizations, government, non-profit organizations, and local businesses were suggested, perhaps coordinated and facilitated by the community sport development structure suggested previously. In particular, panellists suggested linking or partnering with workplace sport initiatives, community and health initiatives for physical activity, and other events. These partnerships could provide opportunities for more integrated community-wide goal setting, while extending the resources for each organization through resource sharing (e.g., volunteers, publicity, facility use). 
Event organizations. Several challenges specific to event organizers were identified via the workshop. One set of challenges is a function of the temporary nature of most event organizations - issues of knowledge transfer and leveraging in the post-event period when the event organization shrinks or ceases to exist. The other set relates to event ownership and requirements set by the event owners through bid documents. Knowledge management was seen as challenging for event organizations. There was a sense that event organizations, in an attempt to be 'the best event,' refuse to share key planning and leveraging knowledge accrued in running their own event. This was felt to be problematic for events that move from host community to host community, but was even more challenging for different events within the same community. And yet, coordination among events in the same community was seen to have the greatest potential impact for community sport development.

What coordination currently exists between sport organizations and event organizers tends to be specific to a single sport. For example, national swimming championships would likely bring together the local swim clubs to help with the event, thus opening a channel of communication that could be used to leverage the event to increase local participation in swimming. Yet, coordination between events and between sports opens up the capacity for cross-leveraging and efficiencies accrued through resource sharing. For this to be effective, systems for knowledge sharing are necessary, as are opportunities to develop shared goals and strategies to benefit multiple sports, events, and organizations. The panellists, perhaps optimistically, felt that the creation of a community-wide sport development organization would serve this function. The partnerships and shared organization would also assist event organizers to leverage in the postevent period by providing an on-going entity to coordinate leveraging efforts. Partnerships with 
the city's tourism entities and city marketing arm were identified as potentially useful partnerships for post-event leveraging.

The second set of challenges reflects the realities of bidding and organizing events owned by other entities. Specific challenges identified were: (a) limitations specified in bid requirements, (b) category exclusivity of sponsorships, and (c) lack of opportunities for local businesses to be official partners. These challenges reflect more traditional thinking about event-business relationships, which tends to see businesses in terms of the resources they can provide to events particularly through formal sponsorship opportunities. Event leveraging, however, provides opportunities for local businesses to have a longer-term relationship with the event and its other partners (e.g., sport organizations). Importantly, within the leveraging context these relationships can occur outside of the official sponsorship rules specified by event owners, including category exclusivity. While some would argue that this is a form of ambush, this strategy provides local businesses with opportunities that would not otherwise be available to them. At the same time, it assists sport organizations to develop potentially long-term relationships with businesses and helps all entities by providing needed resources to the leveraging effort to enhance sport participation and development.

Sport organizations. The challenges facing sport organizations fall into two categories: (1) general challenges clubs face in recruiting and retaining participants that may be exacerbated by the event, and (2) challenges resulting from the event itself. Events and the attention provided by the event can provide a competitive advantage to the sport(s) highlighted by the event. Since sport clubs already feel that they compete for the same athletes, leveraging for participation in a particular sport can create disparities that make it more difficult for clubs to work together - either within a sport or across sports. Leveraging efforts that provide a community focus and goals for 
sport development, and on-going cooperation among sport clubs (and others) to the benefit of all can do much to showcase the benefits of cross-leveraging and cross-promoting sports. In the longterm, this could begin to change the culture of distrust and competition between clubs.

The event itself gives rise to two challenges identified by the panel. Major events are often accompanied by investment in building or refurbishing facilities. These new facilities may increase prices, thus affecting sport clubs' ability to rent space thereby reducing the number of participants clubs can serve. When rental rates do not initially increase, there is a tendency to crowd the new facility with additional clubs. The combination of attention on the sport and the attractiveness of a new facility creates increased demand for the facility as well as for training and club services. Further price increases can result. Perhaps more importantly, the demand for sport services may exceed clubs' structural capacity to serve interested participants. This challenge can result in poor experiences, short-term increases in participation followed by sharp decreases, and even loss of existing members due to decreases in service quality. Panellists emphasized the need to project future needs and build providers' capacity prior to an event in order to avoid becoming a victim of one’s own leveraging success.

Non-sport organizations. Panellists identified challenges for leveraging sport participation and development within systems and structures that may have a stake in, but are not directly linked to the event or with sport in general. From an educational perspective, panellists saw the key challenge for schools to be their overriding mission and focus on academic curricula, which marginalizes the importance of physical literacy and consequently the motivation for sport participation. Since schools have significant reach and contact with participant markets, effective leveraging strategies would benefit greatly from partnerships with schools. These partnerships would enable communication of opportunities (both sport- and event-related) across the entire 
school-aged population. The partnership could be further enhanced by developing programs for school-aged children with specific educational content related to the event, its sport(s), the teams and athletes, as well as the countries, regions, and states represented.

Government involvement was generally seen as a useful tool for leverage. However, regime change and the resultant effects on political agendas and priorities were identified by panellists as particularly challenging to local government and quasi-government entities' ability to leverage events for sport development. Strategies to require sport participation objectives as part of hosting requirements were suggested by the panellists as one way to overcome challenges affecting priorities for sport development. In particular, panellists felt that sport participation objectives should be tied to funding structures such that funding would be contingent on meeting sport participation goals. This would strengthen the impetus for leveraging events for sport development outcomes.

Business involvement in event leverage was largely discussed in terms of sponsors. As with other structural elements, panellists articulated the need for cooperation. It was suggested that leveraging for sport participation could become a tool for sponsors to activate their sponsorship. For example, a cycling equipment manufacturer sponsoring the World Cycling Championships could work with sport and event organizers to sponsor community clinics and workshops leading up to the event. These community events would provide opportunities for the sponsor to leverage their event sponsorship with local area cyclists while stimulating increased participation and product consumption.

Challenges and strategies that emerged from the workshop with expert panellists were overwhelmingly focused on structures and systems. Since most worked within those structures and systems, this is not surprising. In fact, it is encouraging since this level represents the most likely 
level to effect change. The necessary questions to formulate leverage with reference to structures and systems are provided in Table 3.

\section{Resources}

Resources were seen to be critical across all areas of leverage and for each of the organization types. The panel of experts specifically identified three types of needed resources: (1) physical resources, (2) human resources, and (3) knowledge. Financial resources were considered necessary, but only in terms of the ability to purchase or develop resources in the foregoing three areas. Thus, financial resources could be necessary for obtaining one or more of the three core resources if those were lacking, but they were nonetheless insufficient for leverage to take place. Leverage ultimately requires appropriate application of resources to formulate and implement leveraging strategies and tactics.

Physical resources. It is commonly accepted that events can usurp physical resources necessary for sport participation (i.e., facilities and equipment) during an event, but these resources are also limited in the lead up to an event. This shifting of physical resources pre-event and crowding out of physical resources during events takes away sporting opportunities for existing participants, and all but ensures that new participation opportunities are scarce or non-existent. Ironically, the facilities and equipment needed just to maintain local sport participation are scarcest just when media attention and leveraging efforts have their best chance to encourage increased sport participation.

This challenge can be addressed in different ways. First, the problem needs to be identified and acknowledged; subsequently, value-added alternatives can be offered. For example, if a basketball team cannot use its regular facility, another facility can be arranged for them as a suitable alternative, or a different time slot can be provided. In some cases, post-event promotions 
may be necessary to bring participants back to their initial programs. For example, the training period could be extended, or additional training sessions can be offered at no extra cost. Another option is to create temporary facilities to host the event, so that regular programming is not affected. Alternatively, cross training of participants can be considered. For example, instead of having a regular practice, provide training opportunities at a gym; or create partnerships with other sports that are not affected by the hosting of the event. In cases where existing facilities are refurbished or new facilities are built, pro-active planning needs to take place in regards to accessibility and inclusivity.

Retaining existing participants is only part of the challenge. Ultimately, successful event leverage requires that new participants be recruited. To do so, the facilities and equipment must be sufficient and accessible so that the increased demand for sport participation and the variations in skill level that are recruited can be accommodated. This requires projecting the needs and building the necessary capacity prior to hosting the event. It is evident that enhancing provider capacity also includes building capacity in human resources, which will be addressed in the next section.

Human resources. Panellists identified three human resource challenges. The first is to prevent the event from crowding out existing participation in ways that would undermine participation, even by those who already participate. The second is to secure sufficient human resources to serve new participants. The third is to obtain sufficient personnel with appropriate skills to plan and implement leveraging.

All three types of organizations can play a role in addressing each of the challenges. However, event organizers are focused primarily on running the event, and sport is only a secondary concern of non-sport organizations. Thus, the challenge falls primarily to sport organizations to secure and/or train the necessary talent. They may turn to the event organizers 
and non-sport organizations for help where they deem it necessary, but leadership with reference to human resources is theirs.

Events can shift and/or crowd out human resources (i.e., coaches, officials, administrators, volunteers) before and during the event. Again, proactive planning needs to take place to overcome these challenges. Similar strategies as the one proposed for physical resources can be implemented here, namely: (1) identifying/offering value-added alternatives, (2) developing post-event promotions to bring people back, (3) recruiting volunteer coaches, (4) organizing cross-training opportunities for participants, and (5) partnering with other sports.

Successful event leverage that enhances demand will require more human resources post event. Recruiting former athletes as well as family members of athletes, coaches, officials, or volunteers may be one means to build the human resource base. Training current personnel and/or current athletes to take on more responsible roles may be another means to enhance human resources. If financial capabilities enable hiring personnel, then paid staff may also be a solution. The point is that there must be adequate capacity to absorb new athletes into the system if leveraging is to be enabled. Thus, capacities need to be evaluated and augmented where necessary before and during the event.

Having obtained sufficient staff does not assure that leveraging will be enabled or that the sport organization will be able to sustain services to an expanded participant base. In addition to having sufficient labour, that labour must have the time, energy, and skills required to formulate leverage, implement it, and then serve the new participants obtained. That requires more than recruiting new personnel; it requires that those personnel be appropriately trained. That matter is addressed in the next section. 
Knowledge-based resources. Leveraging of any kind is a learned skill. So is sustained management of a sport organization. Workshop participants contended that physical and human resources could only be useful if the people involved in leveraging the event and managing the sport post-event were sufficiently knowledgeable about the event, the overall sport context, management, marketing, grantsmanship, and sponsorship solicitation.

Training from within the sport organization, by the sport's governing body, or through local educational institutions was identified as an obvious means to enhance the knowledge and skills of personnel. It was also suggested that partnerships between or among local organizations could bring knowledge to bear where it might otherwise be lacking. For example, a local sport organization could partner with a local business that is not an official sponsor of the event to develop initiatives that foster sport participation, using the event as a lever. Using current and former athletes may facilitate this task, as local businesses may benefit from the celebrity status of these athletes, and therefore might want to associate themselves with the organizations. Local businesses would be expected to contribute expertise to the leveraging initiative and/or to solicitation of grants and/or sponsorships, thereby bringing their business acumen to the participation development effort.

Just as the quantity of human resources must be sufficient to cope with increased postevent demand, the knowledge, skills, and abilities that personnel bring to managing, coaching, and officiating for new participants are important. New participants must be coached at an appropriate level, and they will also need to learn the subcultural values, beliefs, rituals, and expectations of their sport. Personnel will need to be trained to deliver services appropriately to new participants. That training may need to be solicited from current expertise in the sport organization or from local educational institutions. 
Workshop panellists insisted that training should include (but not be limited to) two topics in particular. To begin, leveraging is at its core a marketing effort. Consequently, panellists felt that media and community relations skills should be strengthened. They also suggested that the necessary grist for media and community relations would be forthcoming if personnel working to market sport participation were trained to identify and use narratives (e.g., athlete stories, event narratives) to create buzz in the community, especially through local media, including social media. The buzz could also create the necessary preconditions for sponsorship and grant solicitation.

The second mandatory topic for training that panellists identified had to do with pathways for participation. They observed that there are multiple settings via which people can take-up a sport. These include sport courses offered by communities, schools, and private organizations. Sports are sometimes offered through workplaces and churches, as well as public or private sport clubs. The pathways by which people can begin a sport, and move vertically or laterally in the sport system should be familiar to those who will solicit and/or work with new participants. Organizations lack suitable metrics to benchmark and evaluate successful leveraging. If leveraging is to be evaluated, which it should, then metrics for that purpose need to be selected and collected. Metrics can include participation rates, club membership rates, number of officials, coaching [levels and numbers], or such aspects as health/wellness, physical activity, attitudes, and volunteer hours, that can be retrieved using social impact measurement tools.

All these efforts take place in a complex policy environment, which includes sport governing bodies as well as local, regional, and national government. In order for a sport to grow, conditions must foster development of physical resources, human resources, and skilful application of knowledge. Therefore, panellists felt that lobbying is also a necessary skill, which should be 
trained and practiced. For these panellists, leveraging sport events requires an array of skills, including soliciting support and building alliances across a diverse array of stakeholders. The necessary questions to formulate resource strategies pursuant to leverage are provided in Table 4. Comparison of the Model with the Legacy of the 1984 Los Angeles Olympic Games

Profits from the Los Angeles Olympic Games were used to endow a foundation to support sport development throughout the southern California region that hosted the 1984 Olympic Games. Initially dubbed the "Amateur Athletics Foundation of Los Angeles," it is now called the LA84 Foundation. In the decades since the close of the 1984 Olympic Games, the foundation has provided grants annually which total more than US\$225 million to support more than 2,200 youth sport organizations serving an estimated 3 million young people (Banks, 2016). In the process, it has supported construction or improvement of nearly 100 sport facilities serving over 500,000 young athletes (Wilson, 2015). In addition, the Foundation provides coach education, and offers a distance running program and a summer aquatics program.

It has been challenging to determine how effective all this has been for developing sport participation insomuch as there were no baseline data from which to track. To evaluate their effectiveness, the LA84 Foundation undertook commissioned a market study of youth (ages 6-17) sport participation rates in Los Angeles County, and compared those to national averages (LA84 Foundation, 2016). For organized sports, participation rates were consistently higher than national averages, and often double or even triple the national average. Outdoor soccer was the most popular, and was played at nearly twice the national average (29\% of LA County respondents vs. $15 \%$ nationally). The two sports in which the LA84 Foundation runs programs directly had participation rates well above national averages: Swimming participation was more than twice the national average (27\% of LA County respondents vs. $13 \%$ nationally), and track and field (aka: 
athletics) was at twice the national average (10\% of LA County respondents vs. $5 \%$ nationally). In contrast to national statistics suggesting declining youth sport participation nationally, $82 \%$ of Los Angeles County youth reported playing at least one sport, and participation rates were high even among adolescents (79\% of females between ages 15-17 and 90\% of males between ages 15-17). Further, over $80 \%$ of those who played sport played more than one sport. Clearly, Los Angeles County has levels of sport participation beyond those generally found in the United States. The LA84 Foundation seems to be making a significant difference.

It is useful to note at the outset that the LA84 Foundation was not envisioned during planning for the Games. Residents of Los Angeles refused to allow their government to subsidize the Games, so the Games were run to enable full cost recovery (Reich, 1986a). This included extensive use of existing sport facilities. In fact, the amount spent on infrastructure by Games organizers in Los Angeles was only 5.4\% of the amount spent for Moscow Olympics infrastructure 4 years earlier, and only $28.4 \%$ of the amount spent for Olympic infrastructure when the Games came to Atlanta twelve years later (calculated from data reported by Wilson, 2015). As a consequence, there was a US $\$ 222.7$ million dollar profit. Under terms of the original contract, a portion went to the IOC, another portion to the USOC, and the remainder was to be dedicated to support the people of southern California, where the Games had been hosted. A Yale law professor, Stanton Wheeler, was recruited to oversee creation of the foundation that would be responsible for those funds. He insisted that those funds be used to support youth sport development in southern California. The initial endowment, which came to US\$93 million, has grown to US\$160 million (Banks, 2016).

This history is important because it highlights three matters. First, in the Los Angeles case, the sport development outcomes were an unforeseen consequence of local politics which had 
insisted that the Games should not tax the public purse, but should instead be run to enable full cost recovery or, if possible, a profit. Second, local politics demanded accountability, which focused attention on the uses to which the money might be put. Third, the persons made responsible for uses of the money insisted that locals should benefit through use of the money to support local sport participation, especially by young people. In fact, youth sport participation was deemed desirable not merely because the Games had been about sport, but also because support for youth sport was acceptable across the political spectrum.

The political feasibility of youth sport support was important because the profitability of the 1984 Olympic Games in Los Angeles came as something of a surprise, which generated substantial political wrangling (Reich, 1984a, 1984b). The political fallout of an unexpected profit rendered the need to identify a politically supportable local purpose for the funds, and to recruit an individual to oversee execution of that purpose. The fact that hosting the Games had been resisted by local taxpayers, elevated the need to find a way to celebrate the financial success of the Games in a manner that would optimize the public relations value and generate widespread political support (cf. Beyette, 1985). A foundation that would share the wealth for sport was the most widely supported political alternative. The system used by the foundation, which requires sport organizations to apply for grants, has nonetheless occasionally frustrated politicians whose districts have not been as well served as others in which sport organizations are more skilled at writing grants (e.g., Reich, 1986b). Thus, local politics have played a role in the foundation's largess since its beginning.

The apparent success of LA84 in supporting youth sport development throughout the region that originally hosted the 1984 Los Angeles Olympic Games is clearly consistent with the model's emphasis on physical resources, as well as its attention to structures and systems. 
Although the structures and systems that eventuated from the Games were unplanned, the presence of substantial funding propelled their creation. Those resources subsequently enabled investment in human resources, including development of local youth sport expertise, as the model mandates. Interestingly, the cultural environment at the time was not one that emphasized sport development, and local attitudes did not support hosting the Games. However the dissonance created by a financially successful Games coupled with the local political wrangling to engender popular opinion and political support for a solution that would direct profits back to the local populace. Since the Games were associated with sport, a sport foundation became the logical means. Although the Games themselves were framed and reported in ideological Cold War terms (Salwen \& Garrison, 1987), those broader political symbolisms of the Games did not play a salient role in planning for use of the Games surplus. Rather, local politics and the need for a visible local benefit dominated planning and directed the outcome. That seems consistent with the model's emphasis on a local focus for event leverage.

The salient difference between the LA84 experience and the experts' model is that the experts sought means to incorporate the event into marketing for participation throughout the hosting period (before and during the event); whereas LA84 used the Games' surplus to do so afterwards. This finding suggests the vital role that resources play in the sustainability of promoting participation from an event, and it suggests that those resources can be useful beyond the period of the event.

\section{Discussion}

The model illustrates a holistic approach to event leverage for purposes of enhancing sport participation, and it provides detailed guidelines for formulation and implementation of the necessary strategies and tactics. Panellists were clear that leverage is not something that can be 
undertaken solely by one organization or another. Event organizers are so focused on running their event that responsibility for leverage is an added burden they can rarely shoulder; sport organizations require support from event organizers to facilitate leveraging; event organizers and sport organizations need assistance from non-sport organizations. Consequently, leverage requires alliances among organizations if it is to be effective. Although this necessity has been noted in previous work on social (Chalip, 2006) and economic (Chalip, 2004) event leverage, it has been understated. Strategic leverage calls for strategic cooperation among stakeholders. More research is needed to identify the necessary means and conditions for forming, sustaining, and utilizing the requisite alliances for leverage.

The complex array of matters to be addressed in order for a sport event to enhance sport participation may explain why research has so far found no systematic positive participation benefit from sport events. It is unlikely that a sufficient array of requisite conditions could or would eventuate without sustained purposeful effort. The expert panellists contended that sport events could be used to build sport participation, but only if events were incorporated into a comprehensive strategy the builds sport events into sport organizations' overall marketing mix. So far, that has rarely been forthcoming, as events have been expected to generate participation simply through the demonstration of sporting competition. While the demonstration that events provide may be facilitative, it is by no means sufficient. Further work is required to identify factors that enable and that hinder effective incorporation of sport events into marketing for sport participation.

In fact, panellists were clear that events and demonstrations of sporting excellence could have negative effects on sport participation. When an event crowds out the time and space (especially in quality facilities) that local participants seek in order to engage in their sport, they may become discouraged and choose to drop out, reduce their level of participation, or find 
alternative recreational activities. If an event attracts elite athletes whose performances seem extraordinary, excellence may seem unattainable to those who observe those performances. In that instance, they may decide that participation is not for them. Research to date has focused on the potentially inspirational effects of elite sport, particularly as showcased through events. Potentially negative aspects of events and elite sport performances need further study, and the means to counter any such effects need to be identified.

The matter of capacity is also important, but too often overlooked. Added participation requires that there be sufficient additional coaching, officiating, equipment, and facility space available to absorb new participants. Unused capacity is inefficient, so at the time an event occurs, it is reasonable to expect that the amount of capacity available to absorb new participants will be small or nonexistent. In that case, it is unlikely that an event could generate new participation, even if demand were stimulated. For that reason, a necessary prerequisite for event leverage will be capacity development. Evaluation of existing capacity, forecasting the added capacity that can be expected from leveraging an event, and then building the necessary capacity are necessary steps in the process. Indeed, it is possible that insufficient unused capacity has played a role in past failures of events to foster sport participation. More work is needed to develop forecasting models that accurately predict sport demand that can be generated by leveraging an event, and the most effective and efficient means to create the capacity that will be needed to meet that demand.

Nevertheless, effective leveraging requires collective action. The panellists felt that a shared strategic vision is a perquisite for any leverage. The model presupposes that there is sufficient commitment among stakeholders that goals will have been set so that a strategy can be formulated and implemented using the guiding questions identified here. The means to initiate the necessary collective action were not addressed by the panellists. Although the challenges for 
creating and sustaining collective action have been much studied (Medina, 2009), the necessity of collective action when seeking outcomes from events has been ignored by researchers and by event owners. The current fashion is to speak in terms of event legacies, and then to place responsibility for obtaining those legacies into the hands of event organizers. Indeed that is often a requirement of event bids. Despite the fact that it is unrealistic, the practice is sustained because it maintains the proprietor-to-client relationship that event owners prefer to have with event organizers (MacAloon, 2008). Current research and theory in collective action may provide constructive alternative insights, and may be usefully tested in the context of research into strategic leverage of sport events.

The Los Angeles case is consistent with the general terms of the model, and highlights the need for event planning that enables resources to be developed and distributed. Although sport development was not initially planned as a Games legacy, it did result from event planning and political forces that enabled and then mandated sustainable sport development outcomes.

Although the foci of the expert model and the LA84 experience are comparable, there is one important difference. The model assumes that the event will be incorporated into local marketing for sport participation. That did not happen in Los Angeles. Rather, the effort took place after the event because it was enabled by windfall profits from the Games. This suggests the utility of the model beyond the period of an event, and it recommends that financial planning should include a plan for creation and use of a financial surplus.

From a sport development standpoint, it is somewhat surprising that the lessons of Los Angeles have not been carried forward to subsequent mega-sport events. Indeed, the direction for mega-sport events has been quite different, as bidding criteria and facility construction have since been inconsistent with the Los Angeles example (Weiss, 2015). It is not clear whether application 
of the model recommended by panellists in this study can fully compensate for the current direction that mega-event hosting has taken, although it does suggest a framework for endeavouring to do so. The Los Angeles example remains a distinctive example of what might be possible.

There have, however, been government-initiated efforts to compel grassroots sport development in concern with sport events. Yet a top-down approach to sport development may be counterproductive insomuch as it fails to take adequate account of local conditions, and it privileges the wants and needs of sport organizations at the top of the sport apex rather than at the grassroots (Harris \& Houlihan, 2016). In that regard, the Los Angeles example stands in stark contrast, as it has never been a government-run organization; nor is it at the apex of the sport system. Rather, it was created and is run as a local initiative with a specific responsibility for enabling local sport organizations to develop their sports.

Although the Los Angeles example suggests what might be achieved through a long-term plan that builds local development initiatives from successful implementation of a large sport event that does not imply that it can be copied elsewhere. The panellists suggest that leveraging participation requires strategies and tactics that are tailored to the conditions in which they will be applied. The local level planning, long-term implementation, and realms of engagement that they recommend are consistent with the Los Angeles example. Nevertheless, the Los Angeles example remains unique, and the broader application of strategic planning that the panellists recommend requires further exploration through application. Action research that applies and evaluates the model developed here will do a great deal to advance our understanding of the strategies, tactics, and challenges of event leverage - especially the ways that variations in local conditions mandate tailored strategic planning.

Acknowledgment: This work was supported by the Social Sciences and Humanities Research Council of Canada/Sport Canada Research Initiative under grant \#862- 2010-0006. 


\section{References}

Attali, M. (2016). The 2006 Asian Games: Self-affirmation and soft power. Leisure Studies, 35, 470-486.

Banks, S. (2016, April 12). What did L.A. get from the '84 Games? An Olympic-size boost for kids. Los Angeles Times. Retrieved April 30, 2016 from http://www.latimes.com/local/california/la-me-banks-olympic-swim-20150822column.html.

Beyette, B. (1985). Los Angeles reflect on legacy of the Olympics: City sees itself in new light a year after flame is extinguished at Coliseum. Los Angeles Times, pg. F2, F21.

Brookes, S., \& Wiggan, J. (2009). Reflecting the public value of sport: A game with two halves. Public Management Review, 11(4), 401-420.

Carter, T. F. (2016). Cuba's challenges hosting the 1991 Pan-American Games and the spectacle of the revolution's ‘soft power'. International Journal of the History of Sport, 33, 186-202.

Cashman, R. (2010). Paradise of sport: A history of Australian sport. Petersham, NSW: Walla Walla Press.

Chalabaev, A., Sarrazin, P., Fontayne, P., Boiche, J., \& Clement-Guillotin, C. (2013). The influence of sex stereotypes and gender roles on participation and performance in sport and exercise: Review and future directions. Psychology of Sport and Exercise, 14, 136-144.

Chalip, L. (1987). Multiple narratives, multiple hierarchies: Selective attention, varied interpretations, and the structure of the Olympic program. In S-P. Kang, J. MacAloon, \& R. DaMatta (Eds.), The Olympics and cultural exchange (pp. 539-576). Seoul: Hanyang University Institute for Ethnological Studies. 
Chalip, L. (1992). Construction and use of polysemic structures: Olympic lessons for sport marketing. Journal of Sport Marketing, 6, 82-98.

Chalip, L. (2001). Group decision making and problem solving. In B.L. Parkhouse (Ed.), The management of sport: Its foundation and application (3rd ed.). Boston: McGraw-Hill.

Chalip, L., \& Heere, B. (2014). Leveraging sport events: Fundamentals and application to bids. In I. Henry \& L-M. Ko (Eds.), Routledge handbook of sport policy (pp. 183-194). New York: Routledge.

Costa, C. A. (2005). The status and future of sport management: A Delphi study. Journal of Sport Management, 19, 117-142.

Department for Culture, Media and Sport (2010). Plans for the legacy from the 2012 Olympic and Paralympic Games. London: Author.

Dong, J., \& Mangan, J. A. (2008). Beijing Olympics legacies: Certain intentions and certain and uncertain outcomes. International Journal of the History of Sport, 25, 2019-2040.

Gibson, O. (2015, March 25), Golden promises of London's 2012 legacy turn out to be idle boasts. Retrieved September 25, 2016 from https://www.theguardian.com/uknews/blog/2015/mar/25/olympic-legacy-london-2012-idle-boasts.

Girginov, V., \& Hills, L. (2008). A sustainable sports legacy: Creating a link between the London Olympics and sports participation. International Journal of the History of Sport, 25, 20912116.

Green, B.C. (2008). Sport as an agent for social and personal change. In V. Girginov (Ed.), Management of Sport Development (pp. 129-146). Oxford, UK: Elsevier. 
Green, M. (2007). Olympic glory or grassroots development? Sport policy priorities in Australia, Canada and the United Kingdom, 1960-2006. International Journal of the History of Sport, 24, 921-953.

Grix, J., \& Brannagan, P. M. (2016). Of mechanisms and myths: Conceptualising states' "soft power" strategies through sports mega-events. Diplomacy \& Statecraft, 27, 251-272.

Grix, J., \& Carmichael, F. (2012). Why do governments invest in elite sport? A polemic. International Journal of Sport Policy and Politics, 4, 73-90.

Grix, J., \& Houlihan, B. (2014). Sports mega-events as part of a nation's soft power strategy: The cases of Germany (2006) and the UK (2012). British Journal of Politics \& International Relations, 16, 572-596.

Harris, S., \& Houlihan, B. (2016). Implementing the community sport legacy: The limits of partnerships, contracts and performance management. European Sport Management Quarterly, 16, 433-458.

Hart, B. H. L. (1991). Strategy ( ${ }^{\text {nd }}$ Rev. Ed.). New York: Plume.

Harvey, N., \& Holmes, C. A. (2012). Nominal group technique: An effective method for obtaining group consensus. International Journal of Nursing Practice, 18, 188-194.

Hindson, A., Gidlow, B., \& Peebles, C. (1994). The ‘trickle down' effect of top-level sport: Myth or reality? A case study of the Olympics. Australian Leisure and Recreation, 4(1), 16-24.

Hong, E. (2011). Elite sport and nation-building in South Korea: South Korea as the dark horse in global elite sport. International Journal of the History of Sport, 28, 977-989.

Jay, K. (2013). More than just a game: Sports in American life since 1945. New York: Columbia University Press.

Koch, N. (2013). Sport and soft authoritarian nation-building. Political Geography, 32, 42-51. 
LA84 Foundation (2016). LA84 Foundation youth sports survey: Los Angeles County, 2016. Los Angeles: Author.

Levitt, T. (1983). The marketing imagination. New York: Free Press.

MacAloon, J. J. (2009). 'Legacy' as managerial/magical discourse in contemporary Olympic affairs. International Journal of the History of Sport, 25, 2060-2071.

Medina, L. F. (2009). A unified theory of collective action and social change. Ann Arbor, MI: University of Michigan Press.

Misener. K., Harman, A., \& Doherty, A. (2013). Understanding the local sports council as a mechanism for community sport development. Managing Leisure, 18(4), 300-315.

Misener, L. (2013). Events and social capital. In R. Finkel, D. MacGillivray, G. McPherson, \& P. Robinson (Eds.), Research themes for events (pp. 18-30). Oxfordshire, UK: CABI.

Mitrofff, I. I., \& Betz, F. (1972). Dialectical decision theory: A meta-theory of decision-making. Management Science, 19(1), 11-24.

MORI (2004). The sports development impact of the Commonwealth Games 2002. Manchester, UK: Author.

Mules, T., \& Faulkner, B. (1996). An economic perspective on special events. Tourism Economics, 2, 107-117.

Parks and Recreation Ontario (2014). About us. Retrieved from http://highfive.org.

Putsis, W. (1998). Economic impact analysis versus cost benefit analysis: The case of a mediumsized sport event. Journal of Macromarketing, 18, 24-33.

Reich, K. (1984a). Olympic surplus grows - as does pressure to share. Los Angeles Times, pg. 1. Reich, K. (1984b). Olympic surplus soaring: Nears \$200 million; Many seeking to share. Los Angeles Times, pg. 1. 
Reich, K. (1986a). Making it happen: Peter Ueberroth and the 1984 Olympics. Santa Barbara, CA: Capra Press.

Reich, K. (1986b). Upset at handing of Olympic surplus: Miffed L.A. councilmen delay funds due to LAOOC. Los Angeles Times, pg. A1, A21.

Reis, A. C., Rodrigues de Sousa-Mast, F., \& Gurgel, L. A. (2013). Rio 2016 and the sport participation legacies. Leisure Studies, DOI: 10.1080/02614367.2012.758304

Ritchie, J. R. B. (2000). Turning 16 days into 16 years through Olympic legacies. Event Management, 6(3), 155-165.

Salwen, M. B., \& Garrison, B. (1987). Sports and politics: Los Angeles Times coverage of the 1984 Summer Olympic Games. Newspaper Research Journal, 8(2), 43-51.

Spender, J-C. (2014). Business strategy: Managing uncertainty, opportunity, and enterprise. New York: Oxford University Press.

SportScotland (2004). Curling success and its impact on participation. Glasgow: Author.

Taks, M., Green, B.C., Misener, L., \& Chalip, L. (2014). Evaluating sport development outcomes: The case of a medium sized international sport event. European Sport Management Quarterly. DOI: 10.1080/16184742.2014.882370

Taks, M., Kesenne, S., Chalip, L., Green, B. C., \& Martyn, S. (2011). Economic impact analysis versus cost benefit analysis: The case of a medium-sized sport event. International Journal of Sport Finance, 6, 187-203.

Taks, M., Misener, L., Chalip, L., \& Green B. C. (2013). Leveraging sport events for participation. Canadian Journal for Social Research (Recent Research on Sport in Canada), 3(1), 12-23. 
Toohey, K. (2008). The Sydney Olympics: Striving for legacies - Overcoming short-term disappointments and long-term deficiencies. International Journal of the History of Sport, 25, 1953-1971.

Veal, A. J. (2003). Tracking change: Leisure participation and policy in Australia 1985-2002. Annals of Leisure Research, 6, 245-277.

Weiss, S. R. (2015). Peter Ueberroth's legacy: How the 1984 Los Angeles Olympics changed the trajectory of the Olympic Movement. International Journal for the History of Sport, 32, $157-171$.

Wilson, W. (2015). Sports infrastructure, legacy and the paradox of the 1984 Olympic Games. International Journal of the History of Sport, 32, 144-156. 


\section{Table 1: Strategic Culture Questions}

1. How can we optimize the buzz around the event, especially for target market segments?

a. What narratives can we build? How can we disseminate them?

b. How can we incorporate and use festival?

c. What special and targeted activities might we add to the event to engage target segments?

d. How can symbols be layered to enhance a sense of meaning for target segments?

2. How can we create and use ancillary events (e.g., road shows, festivals) before, during, and after the event in order to extend the event's reach to target segments, and the frequency of its messaging to those segments?

3. How can the non-sport sector help to enhance the salience of the event and its sport(s), especially for so-called minor sports? 
Table 2: Strategic Questions about Attitudes and Opinions

\section{Event Organizers}

1. How can we structure and design ceremonies so that they will be most useful for shaping attitudes and opinions in ways that can establish optimal conditions from which to leverage the event for sport participation?

2. How can we design and embed festivals to enhance uses of the event to shape attitudes and opinions in ways that can establish optimal conditions from which to leverage the event for sport participation?

3. What can we do before, during, or after the event to help potential athletes (i.e., those who are not yet participating in the sport) to identify with the event's elite athletes? What will minimize any sense of emotional and/or talent distance?

4. What can we do before, during, or after the event to help fledgling athletes (i.e., those who are participating in the sport, but still new to it) to identify with the event's elite athletes? What will minimize any sense of emotional and/or talent distance?

\section{Sport Organizations}

5. How can we use the event to provide attractive opportunities for non-participants to try the sport?

6. How can we use the event to provide information (beyond product trial) about the sport that will be interesting and engaging for non-participants and/or new participants? 
Table 3: Strategic Questions about Structures and Systems

Community Level

1. What are our goals for sport participation? What are our target market segments? How do our goals vary by segment, if at all?

2. What portfolio of events can be brought to bear on our efforts to build sport participation?

3. Which organizations need to be involved for us to achieve our goals?

4. How can those organizations be recruited into the effort? How can they then be coordinated?

5. What resources need to be brought to bear? Which can be provided by organizations involved in the effort? Which need to be obtained elsewhere (e.g., government, sponsorship)?

\section{Event Organizers}

6. How can sport events in the host community's events portfolio be cross-leveraged to enhance the overall leveraging effort?

7. How can knowledge be managed and transferred between local events and for succeeding years in order to optimize leveraging?

8. What organizations need to be brought into alliance to enable leverage? Who should represent those organizations? How should they be incentivized to participate?

\section{Sport Organizations}

9. What capacities and/or skills are required in order for the sport organization to leverage the event to build participation? How can those capacities and/or skills be obtained?

10. What are the potential effects of the event (especially any facility changes) on availability of space and/or time for sport programs? How can those be addressed where needed to minimize negative effects on participants and their experience when participating in sport programs?

11. What are the likely price effects of the event (especially any facility changes)? How can those be addressed to minimize any negative effects on participation?

Non-sport Organizations

12. How can schools help?

13. How can government funding for sport and/or events be provided to foster and/or nurture sport development via events?

14. How can sponsors help to leverage for sport participation?

15. Who else might help with leveraging? 


\section{Table 4: Strategic Resource Questions}

1. Which organizations should be invited and encouraged to join with the social entrepreneur group to form the committee to undertake leverage to build sport participation? Who will be most effective for the leveraging task as a representative of each organization?

2. What training could enhance the effectiveness of the committee undertaking leverage to build sport participation?

3. How crowding out effects of events be redressed or ameliorated?

4. What physical capacities need to be created so that sport program providers can implement and capitalize upon leveraging to build sport participation? How should those be enabled?

5. What human resource capabilities need to be developed so that sport program providers can implement and capitalize upon leveraging to build sport participation? How can those be developed?

6. What training do sport program providers need to enhance their capacity to formulate, implement, and capitalize upon leveraging to build sport participation 


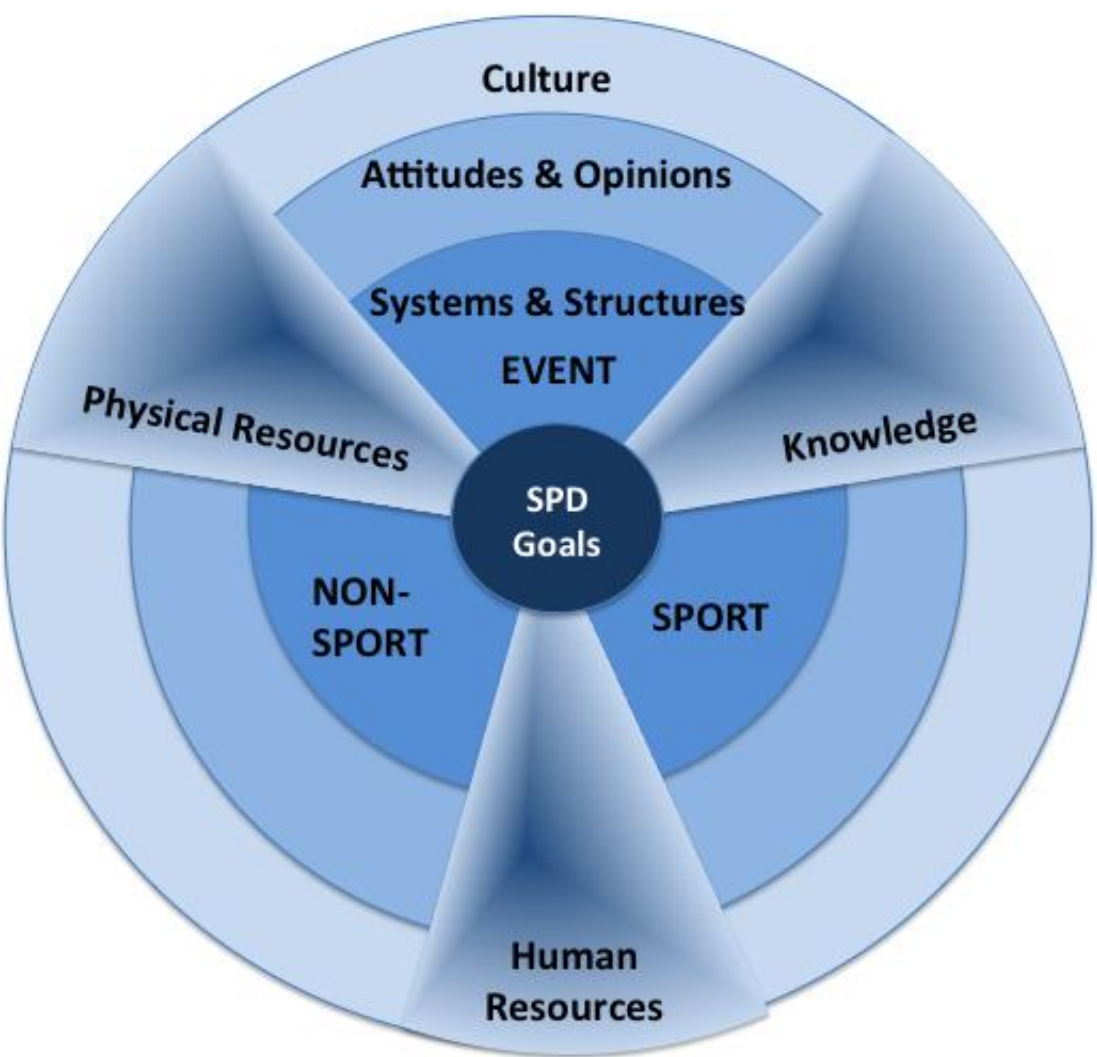

Figure 1: Model for Leveraging Sport Events for Sport Participation and Development 